\title{
Función de los pectenes en detección de presa en hembras del escorpión Centruroides margaritatus (Scorpiones: Buthidae): prueba experimental con grupo control
}

\author{
Jennifer Rivera Hidalgo \\ Instituto Clodomiro Picado, Universidad de Costa Rica. San José, Costa Rica; jennyrih@yahoo.es
}

Recibido 11-IV-2012 Corregido 28-VI-2012 Aceptado 26-VII-2012

\begin{abstract}
Function of pectines for prey detection in female Centruroides margaritatus scorpions (Scorpiones: Buthidae): an experimental test with control group. Scorpions have two mid-ventral organs called pectines, conformed by a row of sensitive tooth-like cylindrical projections. The functions of these appendages are varied, but those related with the detection of preys are controversial. Here, I investigate the prey-detection function of the pectines in the Central American scorpion Centruroides margaritatus. Experimental behavior tests were performed to compare prey detection skills of scorpions with or without alteration of the pectines using an experimental setup. Sixty mature females were assigned randomly to four groups of similar size. The groups include three treatments (application of paraffin, cutting of a pectine and cutting both pectines) and a control group (pectines intact). There was no significant difference in the time of reaction and prey detection between treatments and group control. The frequencies of the behaviors in each treatment differ with those of the control. The modification of the structure suggests a qualitative effect on the capacity to locate prey. The results are discussed in terms of the functionality of the pectines to detect prey. Other receptors (such as trichobothria, tarsal and basitarsal organs) located in pedipalps and legs of the scorpion $C$. margaritatus are possibly more important to perceive substrate-borne vibrations from the prey.
\end{abstract}

\section{KEY WORDS}

Scorpiones, Buthidae, Centruroides margaritatus, pectines, vibrations, prey detection, Costa Rica.

\section{RESUMEN}

La función de los pectenes en escorpiones ha sido objeto de debate por largo tiempo. En general, se sabe que estos órganos están conformados por proyecciones sensitivas cilíndricas que permiten detectar vibraciones y han sido involucrados en la localización de pareja y en el reconocimiento de comportamientos reproductivos de la misma. Una función que ha sido sugerida para los pectenes es la identificación de presas a partir de cambios en las vibraciones del medio. El presente estudio investiga la función de los pectenes del escorpión centroamericano Centruroides margaritatus como estructuras relacionadas a la detección de presas. Pruebas experimentales de comportamiento fueron realizadas para comparar las habilidades de detección de presa en animales con o sin alteración de los pectenes. Sesenta hembras adultas fueron asignadas aleatoriamente a cuatro grupos en números iguales. Los grupos incluyen tres tratamientos (aplicación de parafina, corte de un pecten y corte de ambos pectenes) y un grupo dejado intacto como control. El tiempo de reacción y la detección de presa no difieren entre tratamientos y grupo control. Las frecuencias de los comportamientos observados en cada tratamiento difieren de los controles. La modificación del apéndice sugiere un efecto cualitativo sobre la capacidad para localizar presas. Otros receptores (tricobotrios, órganos tarsales y basitarsales) ubicados en pedipalpos o patas del escorpión C. margaritatus posiblemente sean más importantes para percibir vibraciones emitidas por la presa.

\section{PALABRAS CLAVE}

Scorpiones, Buthidae, Centruroides margaritatus, pectenes, vibraciones, detección de presas, Costa Rica.
La comunicación a través de vibraciones se emplea de diversas formas en el reino animal. Muchos grupos de organismos (mamíferos, reptiles, serpientes) captan información sensorial del medio circundante por medio de vibraciones propagadas en el sustrato (Barnett et al. 1999, O'Connell-Rodwell et al. 2001) o en la superficie del suelo (Young 2003). Esta información puede estar relacionada con alimentación (O'Connell et al. 1997), reproduccióncortejo (Lewis \& Narins 1985), comportamiento antidepredador y alarma (Hill 2001). En el caso particular de artrópodos, las vibraciones son útiles para direccionar la ubicación de presas (Devetak et al. 2007, Mencinger-Vracko \& Devetak 2008), alertar o huir de depredadores (Brownell 1977), así como para localizar y cortejar potenciales 
parejas (Elias et al. 2003). Por otra parte las vibraciones pueden ser empleadas en interacción predador-presa. Fertin y Casas (2007) señalan que las larvas de hormiga león Euroleon nostras (Neuroptera: Myrmeleontidae) utilizan las ondas vibratorias propagadas en la arena para detectar presencia de presas en los túneles trampa. También las orugas son capaces de distinguir en forma cualitativa y cuantitativa las vibraciones provenientes de sus depredadores. Castellanos \& Barbosa (2006) observaron que las larvas de Semiothisa aemulataria (Geometridae) presentan un comportamiento defensivo ante señales emitidas por estímulos vibratorios provenientes de predadores.

Los escorpiones (Arachnida: Scorpiones) son depredadores nocturnos que tienen la habilidad de extraer información proveniente de vibraciones propagadas por sustratos sólidos (Hill 2009). Estos artrópodos poseen numerosos receptores mecánicos y propioreceptores (censan el estado biomecánico) que aumentan la sensibilidad a ondas vibratorias (Blass \& Gaffin 2008). Abushama (1964) sugiere que estos artrópodos detectan las vibraciones a través de tricobotrios, receptores cutilares embebidos en cavidades (botrios), localizados en los pedipalpos. Otros autores como Brownell y Farley (1979 a) sugieren que esto ocurre a través del uso de rendijas sensitivas en las patas. En general los escorpiones muestran gran capacidad para detectar vibraciones e identificar con precisión la fuente de estas. Brownell (1984) argumentó sobre la capacidad de los escorpiones desérticos, para determinar la distancia de presas usando la información proveniente de ondas de compresión tipo (P). Experimentos realizados con el escorpión desértico Smeringurus mesaensis demuestran que esta especie utiliza la información conducida a través del sustrato arenoso para localizar el origen, la dirección y distancia de la fuente de vibración, por medio de sensores mecánicos ubicados en las patas (Brownell \& Farley 1979a, 1979b).

Otra estructura asociada con la percepción de información a través de vibraciones en los escorpiones son los pectenes (Brownell 1988). Estas estructuras son apéndices medio ventrales, se originan desde la superficie ventral cerca de la coxa en los últimos pares de patas (Gaffin \& Brownell 1997, Wolf 2008, Knowlton \& Gaffin 2010). Son consideradas autopomorfías por ser caracteres únicos derivados en el grupo (Polis \& Sissom 1990). Comparten características con el sistema visual y somatosensorial (Root 1990) al detectar características particulares de espacios físicos (Brownell 2001). Brownell (1998) sugiere que las neuronas pectinales conservan la información espacial acerca de la localización de estímulos por proyecciones de neuronas en el ganglio subesofágico. Por otra parte, evidencia morfológica y electrofisiológica demuestran la presencia de receptores de estímulos mecánicos en los pectenes (Melville 2000). Estos receptores median en funciones mecánicas y motoras como el intercambio del espermatóforos durante el cortejo o la detección de cambios en la textura del sustrato. Otras funciones atribuidas a los pectenes incluyen sensibilidad táctil, equilibrio, audición y algunas otras funciones relacionadas con reproducción (Feet et al. 1998).

Las funciones de los pectenes relacionadas con detección y localización de presa son controversiales. Cloudsley-Thompson (1955) reportó que los pectenes detectan las vibraciones del sustrato provocadas por una presa. Abushama (1964) argumentó acerca de la capacidad de los pectenes para monitorear cambios en las características mecánicas y químicas de los sustratos en relación a la presencia o no de presas. Sin embargo Carthy (1968) se opone. Posteriormente Krapf (1986) evidenció la importancia de los pectenes como estructuras accesorias en la detección y localización de presas muertas y sus secreciones, por incremento en actividad de barrido del sustrato. Por otra parte los estudios sobre funciones de pectenes en su mayoría emplean especies desérticas (Swoveland 1978, Gaffin et al. 1992, Gaffin \& Brownell 1992, Knowlton \& Gaffin 2011). Polis \& Sissom (1990) señalan que algunas especies muestran adaptaciones ecomorfológicas a diferentes suelos o microhábitats, sin embargo similitudes en apariencia entre escorpiones solapan las diferencias morfológicas.

El presente estudio utiliza como modelo el escorpión centroamericano Centruroides margaritatus (Gervais 1841) perteneciente a la familia Buthidae, el grupo de escorpiones modernos más numerosos y ampliamente distribuidos. Considerada la especie más abundante en bosques estacionales y caducifolios de Centroamérica (Escobar et al. 2003). En Costa Rica se distribuye desde tierras bajas de boques tropicales secos, en la costa Pacífica hasta el Valle central (1300m) (Víquez 1999). Sin embargo autores como Armas et al. (2011) determinan que las poblaciones presentes desde México hasta Colombia son parte de un taxa restituido y descrito como C. edwardsii (Gervais, 1843). Para efectos de este estudio se mantiene la especie C. margaritatus hasta no darse un cambio oficial en la taxonomía de esta especie para Costa Rica. El objetivo de este estudio fue evaluar la función de los pectenes de C. margaritatus como estructuras relacionadas con detección de presas.

\section{METODOLOGIA}

El estudio se realizó entre los meses de setiembre a noviembre del 2011. Se utilizaron hembras de 7-8cm de longitud del prosoma hasta el aculeus. Los individuos utilizados fueron colectados en el Parque Nacional Santa Rosa, 
Parque Nacional Palo Verde y depositados en la colección viva de escorpiones del Instituto Clodomiro Picado.

Sesenta hembras fueron asignadas aleatoriamente a cuatro grupos en números iguales. Tres grupos fueron designados como tratamientos y uno como control. Cada grupo recibió una larva de Tenebrio molitor (Coleoptera:Tenebrionidae) cada 15 días. Los especímenes se mantuvieron individualmente en cajas de plástico, con múltiples agujeros, algodón húmedo y un trozo de papel tipo kraft como refugio. Las condiciones en el laboratorio se mantuvieron constantes $\left(20-24^{\circ} \mathrm{C}\right.$, una humedad $65 \%$ $75 \%, 12 \mathrm{~h}$ luz).

Los tratamientos aplicados fueron los siguientes: (1) Obstrucción de los pectenes con parafina: se utilizó parafina histológica (Polifin ${ }^{\oplus}$ ), derretida para mejor manipulación. Se colocó una sola capa fina de parafina líquida a temperatura no mayor de $70^{\circ} \mathrm{C}$ con un pincel de punta plana en ambos pectenes. (2) Amputación de un pecten: se amputó el pecten en el punto de unión de la lamela marginal y el pliegue posgenital sin dañar las estructuras reproductoras (Fig. 1). El corte se realizó con una tijera quirúrgica estéril previamente autoclavada. (3) Amputación de ambos pectenes: se amputó ambos pectenes simultáneamente. Los escorpiones en el grupo control permanecieron con sus pectenes intactos, pero también fueron manipulados para causarles estrés similar a los tratados. Todos los individuos se inmovilizaron enfriándolos a $-4^{\circ} \mathrm{C}$ por $3 \mathrm{~min}$. Todos los especímenes comieron una semana antes de aplicar cada tratamiento. Posterior a la aplicación se les dio un período de recuperación de al menos tres días. Después de las pruebas los escorpiones con el bloque de parafina en sus pectenes se les removió manualmente. Todos los individuos fueron utilizados una única vez.

Las pruebas de comportamiento fueron realizadas en una arena experimental cuadrada de plástico con dimensiones de $34,6 \times 21 \times 12,4 \mathrm{~cm}$ rellena con arena de río (Fig. 2). $A$ ambos lados del terrario se ubico una jaula plástica de $3.5 \times 3.5 \times 2 \mathrm{~cm}$ (Fig. 2). La presa (Acheta domesticus) se colocó en la arena experimental en contacto con el sustrato y se alternó la posición de esta (izquierda o derecha) para cada prueba. Antes de iniciar se colocó al escorpión en el centro del terrario (Fig. 2), se inmovilizó con una caja de plástico y se le dio un período de aclimatación de $15 \mathrm{~min}$. Luego se observó el comportamiento durante $20 \mathrm{~min}$. Las pruebas fueron filmadas con una cámara de video digital Sony DCR-HC36 equipada con luz infrarroja. Las grabaciones se realizaron en un cuarto completamente oscuro, simulando el período de actividad de esta especie.

Los comportamientos observados fueron cuantificados en frecuencias cada 30 s durante $20 \mathrm{~min}$. Las variables respuestas analizadas fueron: tiempo de reacción (tiempo que transcurre hasta observar una reacción visible del escorpión), tiempo de encuentro de presa (tiempo que transcurre hasta observar que el escorpión detecte la presa por primera vez). La descripción de los comportamientos descritos se detalla en el Cuadro 1.
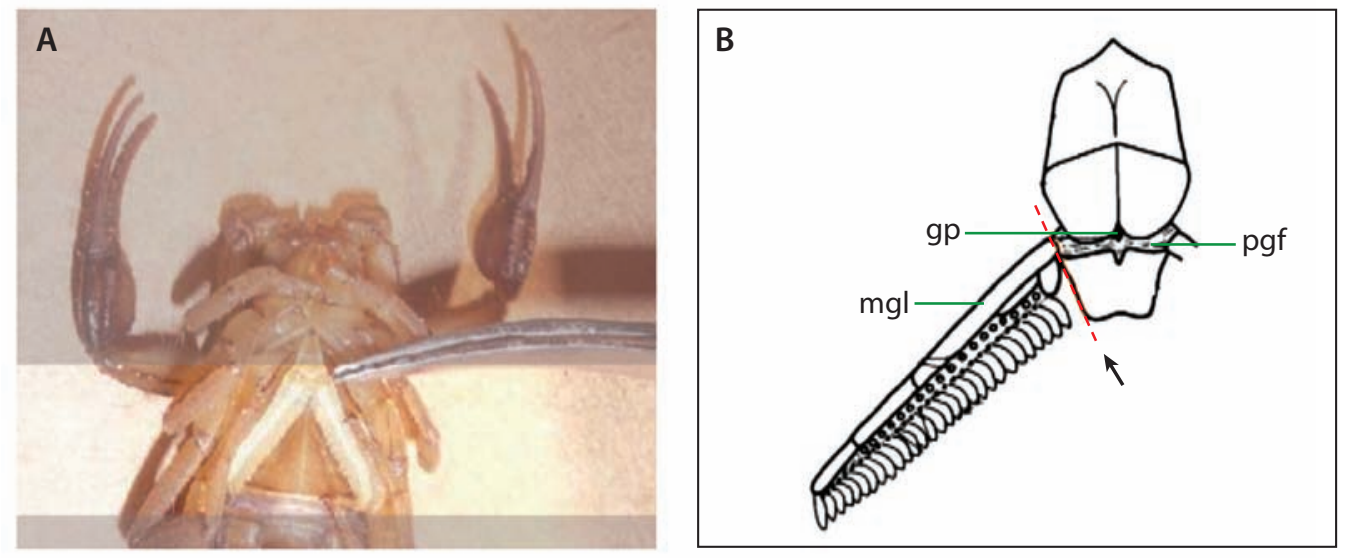

FIG. 1. Morfología del pecten. (A) Pectenes del escorpión localizados en la superficie medio ventral del mesosoma (C. margaritatus). (B) Diagrama de amputación. La flecha indica la dirección de corte; mgl (lamella marginal), gp (papilas genitales), pgf (Pliegue posgenital) (Modificada de Polis \& Sissom 1990). 


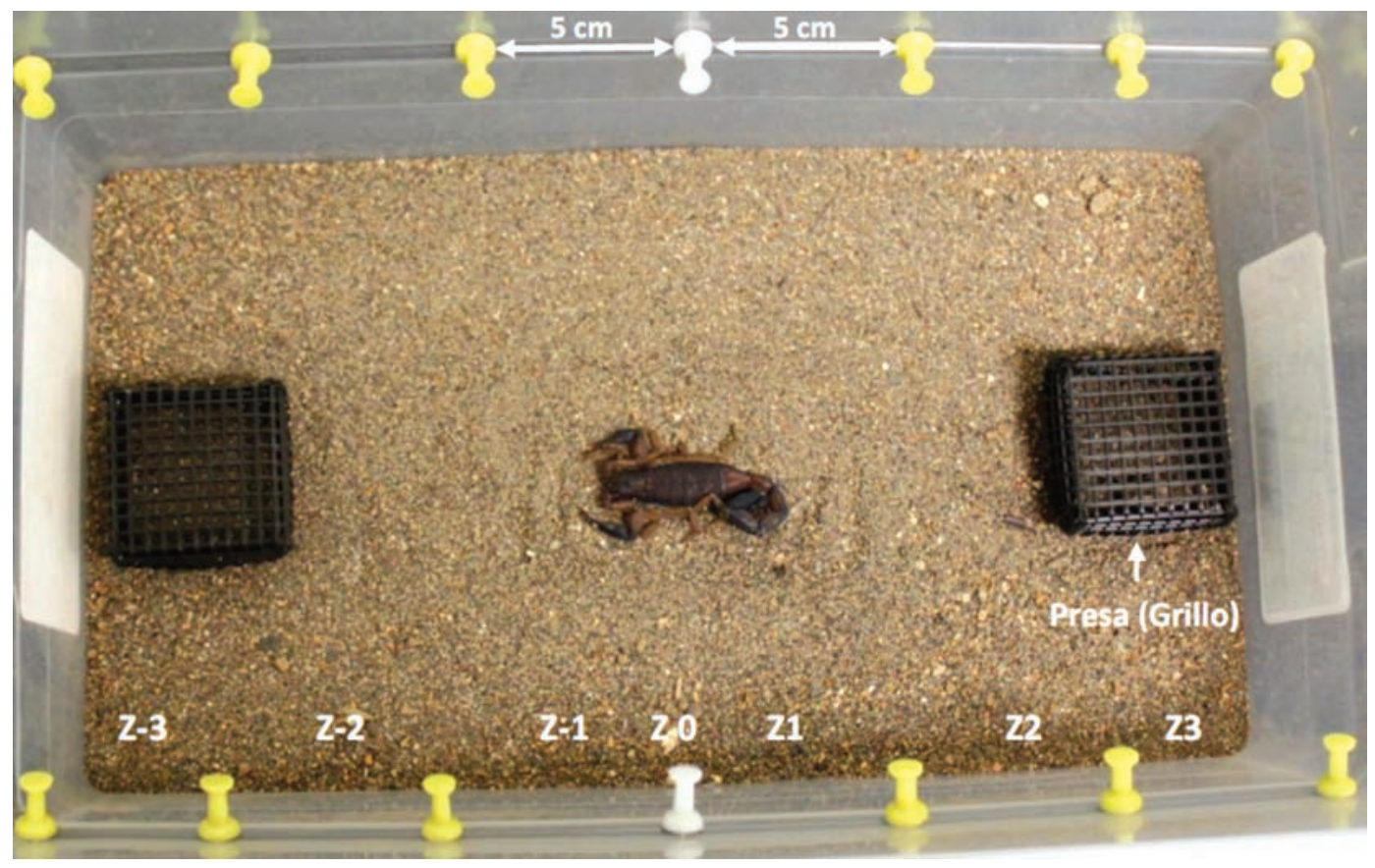

FIG. 2. Arena experimental donde se realizan las pruebas comportamiento. La letra Z corresponde a las zonas de división del terrario, cada zona mide $5 \mathrm{~cm}$ de ancho. Los signos negativos corresponden al lado izquierdo y los signos positivos al lado derecho.

\section{CUADRO 1}

Descripción de los comportamientos observados en hembras de la especie C. margaritatus

\begin{tabular}{ll}
\hline Comportamientos & \multicolumn{1}{c}{ Descripción } \\
\hline Exploratorio & $\begin{array}{l}\text { Caminar de un lado al otro del terrario con los pedipalpos paralelos uno al } \\
\text { otro hacia adelante en forma recta en el mismo plano del cuerpo o haciendo } \\
\text { movimientos hacia arriba y hacia abajo con las tenazas cerradas o abiertas. } \\
\text { Escalar paredes } \\
\text { del terrario }\end{array}$ \\
$\begin{array}{l}\text { Intentar subir las paredes del terrario con los pedipalpos o con ayuda del primer } \\
\text { par de patas apoyando el cuerpo en el abdomen. }\end{array}$ \\
Aseo & Intentar capturar la presa dentro de la jaula plástica, con ayuda de las tenazas. \\
Descanso & Limpieza de tenazas y pedipalpo con quelíceros o con el segundo par de pata. \\
Postura de Alerta & $\begin{array}{l}\text { Cuerpo en contacto con el sustrato, metasoma y apéndices cercanos al cuerpo. } \\
\text { Postura en la cual el escorpión esta soportado encima del sustrato por sus patas, } \\
\text { contacto con el sustrato y metasoma levantado. }\end{array}$
\end{tabular}




\section{Análisis estadístico}

Las proporciones de frecuencias entre tratamientos y grupo control se analizaron con pruebas de chi-cuadrado. Pruebas de Kruskal Wallis se utilizaron para examinar las diferencias en tiempos de reacción y tiempos para hallar presa entre tratamientos y controles. Los análisis estadísticos fueron implementados en el programa JMP ${ }^{\oplus} 7.0$ (SAS Institute 2007).

\section{RESULTADOS}

Los escorpiones incluidos en el tratamiento con parafina, corte de un pecten, corte de dos pectenes y grupo control no difieren en tamaño ( $\mathrm{KW} X^{2}=3,15, \mathrm{gl}=3$, $\mathrm{P}=0,369$ ). Así mismo los tamaños de sus presas (grillos) no difieren ( $K W X^{2}=2,19, \mathrm{gl}=3, \mathrm{P}=0,553$ ). Durante el experimento se observaron cinco categorías de comportamiento (Cuadro 2). El comportamiento más frecuente en el grupo control fue "descanso y limpieza" (28,09\%), "exploratorio $\mathrm{A}^{\prime \prime}$ en los tratamientos uno y dos respectivamente $(46,39 \%$ y $37,29 \%)$, "alerta" (34,45\%) en el tratamiento tres. La frecuencia relativa de todas las categorías de comportamiento se muestra en el Cuadro 2.

La proporción de individuos que respondieron durante los ensayos difiere entre grupos: $93 \%$ en el control, $80 \%$ en los tratamientos dos y tres, y $60 \%$ en tratamiento uno. Estas diferencias son significativas $\left(X^{2}=3,92, g l=1, P<0,05\right)$. El tiempo de reacción, excluyendo a individuos que no reaccionaron no difiere entre tratamientos ( $\mathrm{KW} X^{2}=0,82$ $\mathrm{gl}=3, \mathrm{P}=0,84)$. Así mismo, la proporción de individuos que no lograron encontrar presa durante los ensayos no difiere entre tratamientos y control $\left(X^{2}=2,42, \mathrm{gl}=1 \mathrm{P}>0,05\right)$. El tiempo de encuentro (excluyendo aquellos individuos incapaces de encontrar presa) no difiere entre tratamientos y el control ( $\left.K W X^{2}=3,92, g l=3 P=0,269\right)$.

Las frecuencias totales de las categorías de comportamiento en los grupos tratamiento difieren con respecto al control, Parafina-control $\left(X^{2}=48,02, P<0,0001\right)$, corte de 1 pecten-control $\left(X^{2}=28,01, P<0,001\right)$ y corte de dos pectenes-control $\left(X^{2}=28,01, P=0,001\right)$. Con respecto a la orientación de los individuos al inicio del experimento comparado con el éxito o fracaso de encuentro con la presa para cada tratamiento, no se observan diferencias. La probabilidad de encontrar la presa no depende de estar orientado en dirección a esta inicialmente.

\section{DISCUSIÓN}

La modificación de los pectenes del bútido C. margaritatus tiene un efecto no cuantitativo sobre la capacidad de detección y localización de presas. Pese a que no se observaron diferencias significativas en los tiempos de reacción de los tratamientos, la alteración de pecten parece limitar la precepción de estímulos mecánicos emitidos por la presa (Krapf 1986). Así mismo lo anterior explicaría la variación observada en la proporción de animales que respondieron con el pecten afectado (tratamientos) con respecto a que los que respondieron en el grupo control.

Los datos de este trabajo no indican efecto de tratamiento en cuanto a la frecuencia de individuos que no encontraron sus presas. Diferencias en el éxito de captura por efecto de obstrucción mecánica con parafina, fueron reportados en el escorpión amarillo Tytius serrulatus. Escorpiones con pectenes bloqueados fueron ineficientes

CUADRO 2

Cuantificación de etograma de C. margaritatus en los grupos tratamiento y grupo control

\begin{tabular}{lcccc}
\hline \multicolumn{1}{c}{$\begin{array}{c}\text { Categoría de } \\
\text { comportamiento }\end{array}$} & \multicolumn{4}{c}{ Frecuencia de comportamientos \% } \\
\hline & Parafina & Corte de 1 pecten & Corte de 2 pectenes & Control \\
\hline Exploratorio & 46,39 & 37,29 & 26,72 & 27,01 \\
Escalar paredes & & & 10,65 & 17,71 \\
del terrario & 9,72 & 11,67 & 1,67 & 2,50 \\
Forrajeo & 3,89 & 6,67 & 34,45 & 24,69 \\
Postura de alerta & 14,17 & 20,83 & 26,51 & 28,09 \\
Descanso y limpieza & 25,83 & 23,54 &
\end{tabular}


en percibir, encontrar y capturar presas (Mineo \& Del Claro 2006). En su análisis Mineo y Del Claro (2006) agrupan los especímenes en dos categorías (tratados y controles). Para tres tipos de pruebas los controles muestran la mejor respuesta. Posiblemente la discrepancia con los resultados de estos dos investigadores se deba a heterogeneidad en el grupo control. En mi estudio los individuos del grupo control no siguen el patrón esperado de mejor capacidad de detección de presas.

Los escorpiones tratados detectan la presa de forma similar a los individuos del grupo control. Carthy (1968) reporta que los escorpiones con los pectenes amputados mantienen una respuesta leve a las vibraciones producidas por la presa, debido a la presencia de receptores tarsales y basitarsales ubicadas en las patas, que incrementan la sensibilidad vibratoria. Los receptores basitarsales son activados por ondas en la superficie, mientras que los pelos tarsales responden a ondas de compresión de larga amplitud. Por otra parte investigadores como Brownell y Hemmen (2001) señalan que los estímulos vibratorios en los escorpiones pueden ser censados por rendijas sensitivas embebidas en la cutícula, donde monitorean los cambios en la tensión durante la locomoción. Estos receptores son sensibles a cambios en la frecuencia de ondas mecánicas, por lo cual detectan señales de origen vibratorio debido a la inervación de varias motoneuronas que censan esos cambios. De igual forma, los escorpiones pueden localizar la cercanía de presas vivas con precisión por medio de vibraciones propagadas en el aire (Brownell 1977). Bub y Bowerman (1979) señalan que los tricobotrios funcionan como detectores sensibles a movimiento aéreo que registran las vibraciones aéreas y orientan las acciones de captura. Así mismo estos mecanoreceptores poseen un plano preferencial de oscilación, setas con el mismo plano de deflexión se agregan en grupos y forman un campo receptivo que censa los estímulos provenientes de señales transmitidas a través del aire (Krapf 1986).

Los comportamientos observados en los grupos tratamiento difieren del grupo control en la distribución de frecuencias para cada comportamiento. Las discrepancias posiblemente se deban a un efecto diferencial en la capacidad de cada individuo para realizar un determinado comportamiento. Hölldobler \& Wilson (1990) sugieren que individuos de la misma especie pueden mostrar diferentes reacciones a estímulos similares y los comportamientos ante estos estímulos pueden variar.

Los resultados del estudio muestran un menor porcentaje de "postura de alerta" en aquellos individuos con los pectenes parafinados. La aplicación de la parafina altera los comportamientos de los individuos. Estos resultados se corroboran con los datos obtenidos por Mineo \& Del
Claro (2006), aquellos individuos cuyos pectenes fueron bloqueados mecánicamente presentan menor porcentaje de "postura de alerta", lo cual implica una disminución en el éxito de captura. En contraparte mis datos muestran un mayor porcentaje de individuos con pectenes amputados en "postura de alerta", posiblemente la carencia de los apéndices dificulte la percepción de estímulos. Las neuronas sensoriales que inervan las clavijas sensitivas requieren ser estimuladas con pulsos eléctricos. Estímulos repetitivos con una frecuencia aproximada de $200 \mathrm{~Hz}$ producen un pico mínimo de actividad eléctrica (Root 1990) que permite una sinapsis axónica periferal (Foelix \& Muller-Vorhorlt1983) cuyas aferencias se proyectan topográficamente desde el diente pectinal hasta el sistema nervioso central (Melville 2000). Estas observaciones coinciden con los resultados de Cloudsley-Thompson (1955) que sugieren que la amputación de ambos de pectenes incide en la capacidad de percepción de vibraciones de presas.

En conclusión los pectenes son estructuras que parecen ser importantes en la detección de potenciales presas en Centruroides margaritatus. Autores tales como Foelix \& Müller-Vorholt (1983), Carthy (1966), Gaffin \& Walvoord (2004) Kladt et al. (2007) indican que la presencia de múltiples receptores en el pecten (clavijas sensoriales, pelos pectinales y células mecanorectoras) pueden intervienen en la detección de señales mecánicas que indican la presencia de presas. Por lo que estos receptores parecen potenciar la señal del estímulo vibratorio emitido por una presa y propagado a través del sustrato. Finalmente el papel de los pectenes de C. margaritatus deba evaluarse en conjunto con tricobotrios en pedipalpos, componentes tarsales y basitarsales (rendijas sensitivas) en las patas que posiblemente medien la sensibilidad vibratoria en esta especie.

\section{AGRADECIMIENTOS}

Agradezco al Instituto Clodomiro Picado, en especial a los miembros de la sección del serpentario: Fabián Bonilla, Aarón Gómez, Carmen Vázques R., Erick Ballestero, Jazmín Arias O., Danilo Chacón y Santos Rodríguez por la colaboración durante la realización de los experimentos y préstamo de equipo. Daniel Briceño por su aporte y consejos. A Mahmood Sasa M. por la ayuda en el análisis estadístico y revisión del manuscrito original. Este estudio fue financiado en parte gracias al proyecto 741-A5-048 de la Vicerrectoría de Investigación de la Universidad de Costa Rica. 


\section{REFERENCIAS}

Abushama, F.T. 1964. On the behaviouor and sensory physiology of the scorpion Leiurus quinquestriatus. Anim Behav 12: $140-153$.

Armas, L.F., R., Teruel, \& F., Kovarik. 2011. On Centruroides margaritatus (Gervais, 1841) and closely related species (Scorpiones: buthidae). Euscorpius - Occasional Publications in Scorpiology 132: 1-16

Barnett, K.E., R.B., Cocroft. \& L.J. Fleishman. 1999. Possible communication by substrate vibration in a chameleon. Copeia 1999: 225-228.

Blass, G.R. \& D., Gaffin. 2008. Light wavelength biases of scorpions. Animal Behaviuor 76: 365-373.

Brownell, P.H. 1977. Compressional and surface waves in sand: Used by desert scorpions to locate prey. Science 197: 497-482.

Brownell, P.H. 1984. Prey detection by the sand scorpion. Sci Am 251:86-97.

Brownell, P.H. \& R.D., Farley. 1979a. Detection of vibrations in sand by tarsal sense organs of the nocturnal scorpion, Paruroctonus mesaensis. J Comp Physiol A 131: 23-30.

Brownell, P.H. \& R.D., Farley. 1979b. Orientation to vibrations in sand by the nocturnal scorpion Paruroctonus mesaensis: Mechanism of target localization. J. Comp. Physiol. A 131: 31-38.

Brownell, P.H. 1988. Properties and functions of the pectine chemosensory system of scorpions. Chem Senses 10:557.

Brownell, P.H. 1998. Glomerular Cytoarchitectures in Chemosensory Systems of Arachnids. Annals of the New York Academy of Sciences. Olfaction and taste XII: An international symposium 855: 502-557.

Brownell, P.H. 2001. Sensory ecology and orientational behaviors. 159-183pp. In P. H. Brownell \& G. A. Polis (Eds.). Scorpion Biology and Research. Nueva York, Nueva Yorok, EEUU.

Brownell, P.H. \& J.L., Hemmen. 2001. Vibration sensitivity and computational theory for prey-localizing behavior in sand scorpions. Am Zool 41: 1229-1240.

Bub, K. \& R.F., Bowerman.1979. Prey capture by the scorpion $\mathrm{Ha}-$ drurus arizonensis Ewing (Scorpiones: Vaejovidae). J Archnol 7: 243-253.

Carthy, J.D. 1966. Fine structure and function of the sensory pegs on the scorpion pecten. Experientia 22: 89-91.

Carthy, J.D. 1968. The pectines of scorpions. Symposium of the Zoological Society, London, 23: 251-261.

Castellanos, I. \& P., Barbosa .2006. Evaluation of predation risk by a caterpillar using substrate-borne vibrations. Anim Behav 72: 461-469.

Cloudsley-Thompson, J.L. 1955.On the function of the pectines of scorpions. Ann Mag Nat Hist 8: 556-560.
Devetak, D., B., Mencinger-Vračko, M., Devetak, M., Marhl \& A., Špernjak. 2007. Sand as a medium for transmission of vibratory signals of prey in antlions Euroleon nostras (Neuroptera: Myrmeleontidae). Physi Entom 32: 268-274.

Elias, D.O., A.C., Mason, W.P., Maddison \& R.R., Hoy. 2003. Seismic signals in a courting male jumping spider (Araneae: Salticidae). J Exp Biol 206: 4029-4039.

Escobar, E., L., Velásquez. \& C., Rivera. 2003. Separación e identificación de algunas toxinas del veneno de Centruroides margaritatus (Gervais, 1841) (Scorpiones: Buthidae). Rev. Peru. Biol. 10: 217-220.

Feet, V., G.A., Polis. \& W.D., Sissom. 1998. Life in sandy: the scorpion model. J Arid Environ 39:609-622.

Fertin, A. \& J., Casas. 2007. Orientation towards prey in antlions: efficient use of wave propagation in sand. J Exp Biol 210:3337-3343.

Foelix, R.F. \& G., Muller-Vorhorlt.1983. The fine structure of scorpion sensory organs. II. Pecten sensilla. Bull Brit Arach Soc 6: 68-74.

Gaffin, D.D. \& P.H., Brownell. 1992. Evidence of chemical signaling in the sand scorpion, Paruroctonus mesaensis (Scorpionida: Vaejovidae). Ethology 91:59-69.

Gaffin, D.D., L.W., Kira \& P.H., Brownell. 1992. Water detection in the desert sand scorpion, Paruroctonus mesaensis (Scorpionida, Vaejovidae) J Comp Physiol A. 170: 623-629.

Gaffin, D.D. \& P.H., Brownell.1997. Electrophysiological evidence of synaptic interactions within chemosensory sensilla of scorpion pectines. J Comp Physiol A. 181: 301-307

Gaffin, D.D. \& P.H., Brownell. 1997. Response properties of chemosensory peg sensilla on the pectines of scorpions. J Comp Physiol A. 181: 291-300.

Gaffin, D.D \& M.E., Walvoord. 2004. Scorpion peg sensilla: are they the same or are they different?. Euscorpius - Occasional Publications in Scorpiology. 17:7-15.

Hill, P.S. 2001. Vibration and animal communication: A Review. Amer. Zool.41: 1135-1142.

Hill, P.S. 2009. How do animals use substrate-borne vibrations as an information source? Naturwissenschaften.96: 1355-1371.

Hölldobler, B. \& E., Wilson. 1990. The ants. Harvard University Press, Cambridge, MA

Kladt, N., H., Wolf, \& G.H., Harald. 2007. Mechanoreception by cuticular sensilla on the pectines of the scorpion Pandinus cavimanus. J Comp Physiol A. 193: 1033-1043.

Knowlton, E.D. \& D.D., Gaffin.2010. A new tip-recording method to test scorpion pecten chemoresponses towater-soluble stimulants. J Neurosci Methods 193: 264-27.

Knowlton, E. \& D., Gaffin. 2011 Functionally redundant peg sensiIla on the scorpion pecten. J Comp Physiol 197: 895-902.

Krapf, D.1986. Contact chemoreception of prey in hunting scorpions (Arachnida:Scorpiones). Zoo Anz. 217: 119-129. 
Lewis, E.R. \& P.M., Narins. 1985.Do frogs communicate with seismic signals? Science 227: 187-189.

Melville, J. 2000. The pectine of Scorpion: Analysis of Structure and Function, Oregon State University. Doctoral thesis. Oregon EE.UU.

Mencinger-Vracko, B. \& D., Devetak. 2008. Orientation of the pitbuilding antlion larva Euroleon (Neuroptera, Myrmeleontidae) to the direction of substrate vibrations caused by prey. Zoology 111.1: 2-8.

Mineo, F.M. \& K., Del Claro.2006. Mechanoreceptive function of the pectines in the Brazilian Yellow scorpion Tityus serrulatus: perception of substrate-borne vibration and prey detection. Acta Ethol 9: 79-85.

O'Connell, C.E., B.T., Arnason. \& L.A., Hart. 1997. Seismic transmission of elephant vocalizations and movement. J Acoust Soc Am 102: 3124.

O'Connell-Rodwell, C.E., L.A., Hart. \& B.T., Arnason.2001. Exploring the potential use of seismic waves as a communication channel by elephants and other large mammals. Am Zool 41: 1157-1170.

Polis, G. \& W., Sissom 1990.Life history. The Biology of scorpions. Stanford University California.California.EE.UU.

Root, T.M. 1990. Neurobiology. p 341-413 In: Polis GA (Ed). The biology of scorpions. Stanford University Press, Stanford.

Swoveland, M.C. 1978. External morphology of scorpion pectines. California State University, Master's thesis. San Francisco. EE.UU..

Víquez, C. 1999. Escorpiones de Costa Rica. Scorpions. Instituto Nacional de Biodiversidad (INBio), Santo Domingo, Heredia.Costa Rica 84 p.

Wolf, H. 2008.The pectine organs of the scorpion, Vaejovis spinigerus: Structure and (glomerular) central projections. Arthropod Struct Dev 37:67-80.

Young, B.A. 2003. Snake bioacoustics: toward a richer understanding of the behavioral ecology of snakes. Quart Rev Biol 78: 303-325. 